\title{
A generic model of collectivities
}

\author{
Nigel Gilbert \\ Centre for Research in Social Simulation \\ University of Surrey \\ Guildford \\ United Kingdom \\ n.gilbert@surrey.ac.uk
}

\begin{abstract}
A class of social phenomena, exhibiting fluid boundaries and constant change, called 'collectivities', is modelled using an agent-based simulation, demonstrating how such models can show that a set of plausible micro-behaviours can yield the observed macrophenomenon.
\end{abstract}

Some features of the model are explored and its application to a wide range of social phenomena described.

Running head: A generic model of collectivities

\section{Acknowledgement}

The support of the NewTies project is gratefully acknowledged. The NewTies project is partially supported by the European Commission Framework 6 Future and Emerging Technologies under contract 003752 . Opinions are the author's own and do not represent those of the Commission. 
There are a number of related social phenomena that are hard to model, or even to describe, because their boundaries are fluid, the people involved are constantly changing and there is no single characteristic shared by all those involved. Examples include:

- Youth 'sub-cultures', such as 'punks' (Widdicombe and Wooffitt, 1990), 'goths' (Hodkinson, 2002) or 'Hell’s Angels' (Thornton, 1995);

- Scientific research areas or specialities (Gilbert, 1997);

- Art movements such as the Pre-Raphaelites or the Vorticists (Mulkay and Turner, 1971);

- Neighbourhoods, such as 'Notting Hill' (in London) or the Bronx in New York (O' Sullivan \& Macgill, 2005);

- Members of armed revolutionary movements or 'terrorist' movements (Goolsby, 2006);

- Industrial sectors such as 'biotechnology' (Ahrweiler et al., 2004);

and many more.

Although we can easily point to familiar examples, and although they are very common and easily identified, it is difficult to put one's intuitions about them on a firmer footing. For a start, there is no commonly accepted word with which to name the phenomenon. The terms, sub-culture, area, neighbourhood, specialty and movement are used for particular types, but none of these words are appropriate for describing all of them. Other suggestions might be 'organisation' (but this is usually means a formal organisation with clear boundaries), 'group' (usually a term for a collection of people who all know each other, not necessarily true in all the examples above), or 'institution' (properly used to describe a set of rules, not a collection of people). A closely related concept is Norbert Elias' 'figurations' (Elias, 1939), although this should strictly be applied only to individuals, not to organisations or other types of actor. In what follows, I shall use the term 'collectivity' as the generic term for lack of a better one. 
Note that the units making up the collectivity may be people (as in most of the examples above) or organisations (e.g. biotechnology firms).

A second barrier to gaining a better understanding of collectivities is that, by definition, there is no definite boundary around them (Abbott, 1995). This means that it is impossible to count their members, and therefore to engage in the commoner kinds of quantitative analysis of their development over time, their incidence and so on.

Third, the way in which collectivities arise from the actions of their members is not easily understood. It is the purpose of this paper to suggest how some plausible assumptions about individual action ('micro foundations') could yield the collectivities that are observable at a macro level.

In all collectivities, the following seem to hold, to a greater or lesser extent:

1. While instances of collectivities are usually easily named and described at the aggregate or 'macro' level, precise definitions can prove to be rather slippery and open to negotiation or argument (e.g. there are many slightly different areas that can be described as Notting Hill, from the official local government area to the locality within which the film of the same name was shot);

2. There is no accepted consensual definition that can be used to sort those who are 'in' from those who are 'out' (or members from non-members). For example, while some might think that a person is a 'punk' because of the way that he or she dresses, this assignment might be contested by others (including by the person themselves) by pointing to the person's beliefs, behaviour or acquaintances, all of which could alternatively be relevant for making a decision on membership. In particular, there is no one observable feature which all those who are 'in' and which none of those who are 'out' possess. Collectivities are not, for example, formal organisations (where being an employee with a written or verbal contract distinguishes those who are members) or political parties 
(where at a minimum, a formal declaration of support is required and defines membership), or social classes, where externally specified objective criteria are used to sort people (typically one's occupation).

3. Nevertheless, many of the members will share characteristics in common (e.g. the scientists in a research area may have similar education, have carried out similar previous research, and be known to each other, even if there is no technique, theory or object of research which all of those without exception in the research area are involved with).

4. Membership of the collectivity entails possessing some related knowledge (e.g. the science of the specialty, or whatever is accepted as 'cool' in a youth culture, or the local geography of Notting Hill). However, no member possesses all the knowledge: knowledge is socially distributed.

5. The features that are thought to be relevant to the collectivity change. For example, researchers do not continue to work on exactly the same problems indefinitely; once they have solved some, they move on to new ones, but still within the same research area. Most political movements change their manifestos over time to reflect their current thinking and the social problems that they see around them, although they remain the same movements with many of the same adherents. Youth cultures are constantly changing the items which are considered to be in fashion.

6. Some of the people involved are widely considered (e.g. by the others) as being more central, more influential, of greater status or as leaders as compared with others. For example, some scientists are considered to be more eminent than others; some members of sub-cultures are more 'cool' than the rest and so on.

In the rest of this paper, we first introduce some assumptions about the micro level ('individual') behaviour that might lead to the emergence of collectivities (section 2). We then discuss some related models that might be used to simulate the emergence of collectivities (section 3 ) 
and in section 4 introduce a model which combines some aspects of these. In section 5, we discuss the results of running the model and conclude in section 6 .

\section{Micro-level behaviour}

One of the features common to collectivities mentioned in section 1 is that the actors (i.e. the people or organisations that make up the collectivity) have some special knowledge or belief (e.g. for scientists, knowledge about their research area; for youth sub-cultures, knowledge about what is currently fashionable). Even though this knowledge is socially distributed among the members of the collectivity, so that not every member has the same knowledge, possession of it is often a major feature of the collectivity. In the general model to be introduced in this paper, we assume that all individuals, members and non-members, have some knowledge, but what this knowledge is varies both between actors and over time. We use this knowledge to 'locate' the actors: the abstract 'position' of the actor at a moment in time is a function of the knowledge that he or she possesses at that time.

A second assumption is that some actors are of higher status than others and that all actors are motivated to try to gain status by imitating high status actors (by copying their knowledge). For example, in a collectivity driven by fashion, all actors will want to be as fashionable as they can, which means adopting the clothing style, musical tastes or whatever of those that they perceive to be of the highest status. However, status is also a function of rarity: an actor cannot remain of high status if there are many other actors closely located around him or her (i.e. with very similar knowledge). For example, a fashion icon must always be ahead of the hoi polloi; a scientist will only be heavily cited if the research is distinctive; a revolutionary will only earn the respect of his colleagues if he stands out in comparison with the 'foot soldiers'.

Thirdly we assume that actors who are at the 'leading edge', i.e. are of the highest status, want to preserve this status, which they cannot do if they start to be crowded out by followers 
who have been attracted to them. In this situation, we assume that actors are motivated to make innovations, that is to search out nearby locations where there are not yet crowds.

There are thus two countervailing tendencies for actors: one the one hand they want to get 'close to the action'; on the other, they want to be exclusive and can do so by 'moving the goalposts' that represent the heights of status. As we shall see, working out this tension yields patterns at the macro level that are typical of collectivities.

\section{Related Models}

There are several generic models that deal with similar issues:

a. Boid models (Reynolds, 1987) have agents that try to maintain a desired distance away from all other agents and thus appear to move with coordinated motion. Agents have three steering behaviours: separation, to avoid nearby agents; alignment, to move in the same direction as the average of nearby agents; and cohesion, to move towards the average position of nearby agents. The effect is that agents move as in a flock or school of fish. These models illustrate the effect of having agents carrying out actions that are in 'tension': the separation behaviour is in tension with the cohesion behaviour, for instance. However, there are no notions of seeking status or innovation in these models.

b. Innovation models (Ahrweiler \& Gilbert, 1998) have agents that are able to learn and act according to their current knowledge. Often, agents exchange knowledge, thus creating new knowledge through a process similar to the crossover operator used in genetic algorithms. However, there is no specific idea of collectivity in these models. The set of agents involved in innovation is predetermined.

c. The minority game (Slanina, 2000, is one example from a large literature). This model, also called the El Farol bar model, has agents who wish to go to the bar, but only when a minority of the other agents also choose to go there. The agents make a decision based 
on their own past experience of the number they previously encountered at the bar. Each agent has a number of 'strategies' which they use in combination with their memory of the outcome of recent trips to the bar to make a decision on whether to visit the bar at the current time step. The strategies are scored according to their success (whether, when the agent arrives at the bar, it is overcrowded or not) and unsuccessful strategies are dropped. Over time a dynamic equilibrium can be established, with the number of agents at the bar matching the threshold that agents use to judge that there are too many agents there. This model has some features of the problem addressed in here, but there is no representation of a collectivity.

\section{The Model}

The model described in this section was created using NetLogo version 3.0 and follows the algorithm shown in the Appendix. The code is available from the author and can be run as an applet at http://sandi.soc.surrey.ac.uk/ $\sim \operatorname{scs} 1 \mathrm{ng} / \mathrm{Gencol} /$ genre-6.html.

The model consists of a surface over in which agents are able to move. The surface is a toroid with each point representing one particular set of knowledge or beliefs. The agents thus move, not in a representation of physical space, but rather in 'knowledge space'. While it might be thought to be over-simplifying to represent a 'knowledge space' in two dimensions (more exactly, on the surface of a toroid), it makes for easier visualisation.

An agent's movement in the knowledge space represents its change in knowledge. Thus if an agent imitates another agent, it would be seen to be moving towards that agent in the knowledge space, while if it innovates and discovers knowledge that other agents do not have, it would be seen to be moving away from other agents into previous empty areas of the space.

Agents are initially distributed at random on the surface. Agents have no memory of their own or other agents' previous positions. Each agent: 
1. Counts how many other agents there are in its immediate neighbourhood (i.e. the area surrounding it).

2. If the number is above a threshold, it faces in the average direction that the other agents nearby are going and then moves a random distance in that direction.

3. If the number is equal to or below the threshold, it looks around the locality to find a relatively full area and then moves a random distance from its present location in the direction of that area.

Each agent acts asynchronously, repeating this sequence of actions indefinitely.

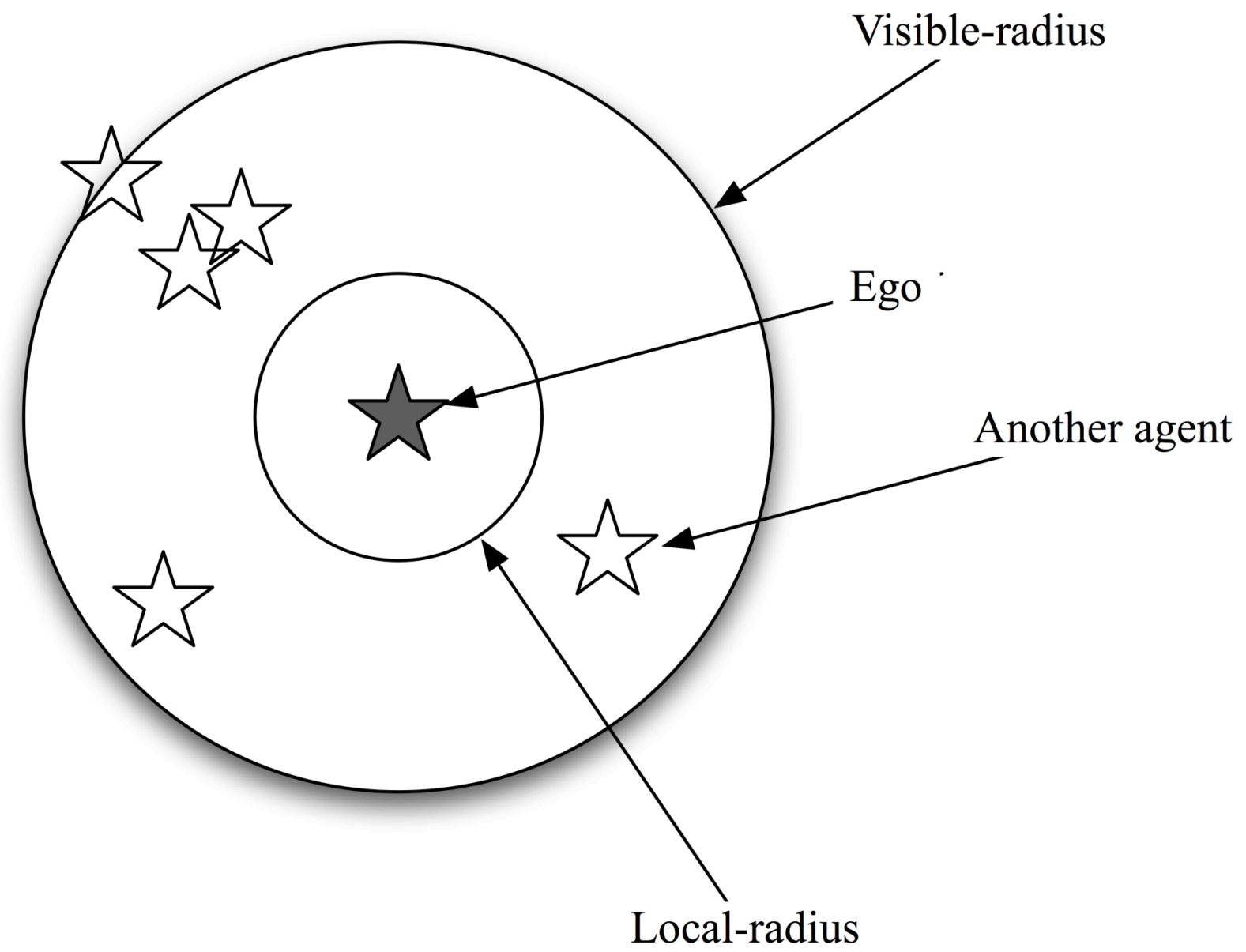

Figure 1: Parameters

There are four parameters required by this algorithm (see Figure 1): 
1. The radius of the circular area surrounding an agent within which the number of agents is counted to determine whether the agent is 'crowded' or 'lonely' (local-radius: default: 3).

2. The threshold number of agents below which the agent is 'lonely' and above which the agent is 'crowded' (threshold: default: 40);

3. The radius of the circular area surrounding an agent in which the agent, if lonely, counts the number of agents to find where there is a maximum or, if crowded, finds the average direction of agent movement in order to determine the direction in which it is to move (visible-radius: default: 10);

4. The distance that an agent moves. The distance is chosen randomly from a uniform distribution with this parameter as the maximum (speed: default: 5).

\section{Results and discussion}

As expected, running the model shows that the initial uniform random distribution of agents separates into 'clumps', in which some agents are central while others are distributed around them. The central agents are crowded, and so move. In doing so, they shift the centroid of the clump slightly and may make other agents either crowded or lonely. Other crowded agents also move, in the same direction as the original crowded agents. Thus the clump of agents, while remaining together for long durations (as measured in time steps), drifts across the surface. Lonely agents move towards the clump, sometimes joining it and sometimes continuing to trail behind it. The clumps never merge.

Figure 2 illustrates a typical snapshot. In this figure, agents that are crowded are dark and those that are lonely are light grey. 


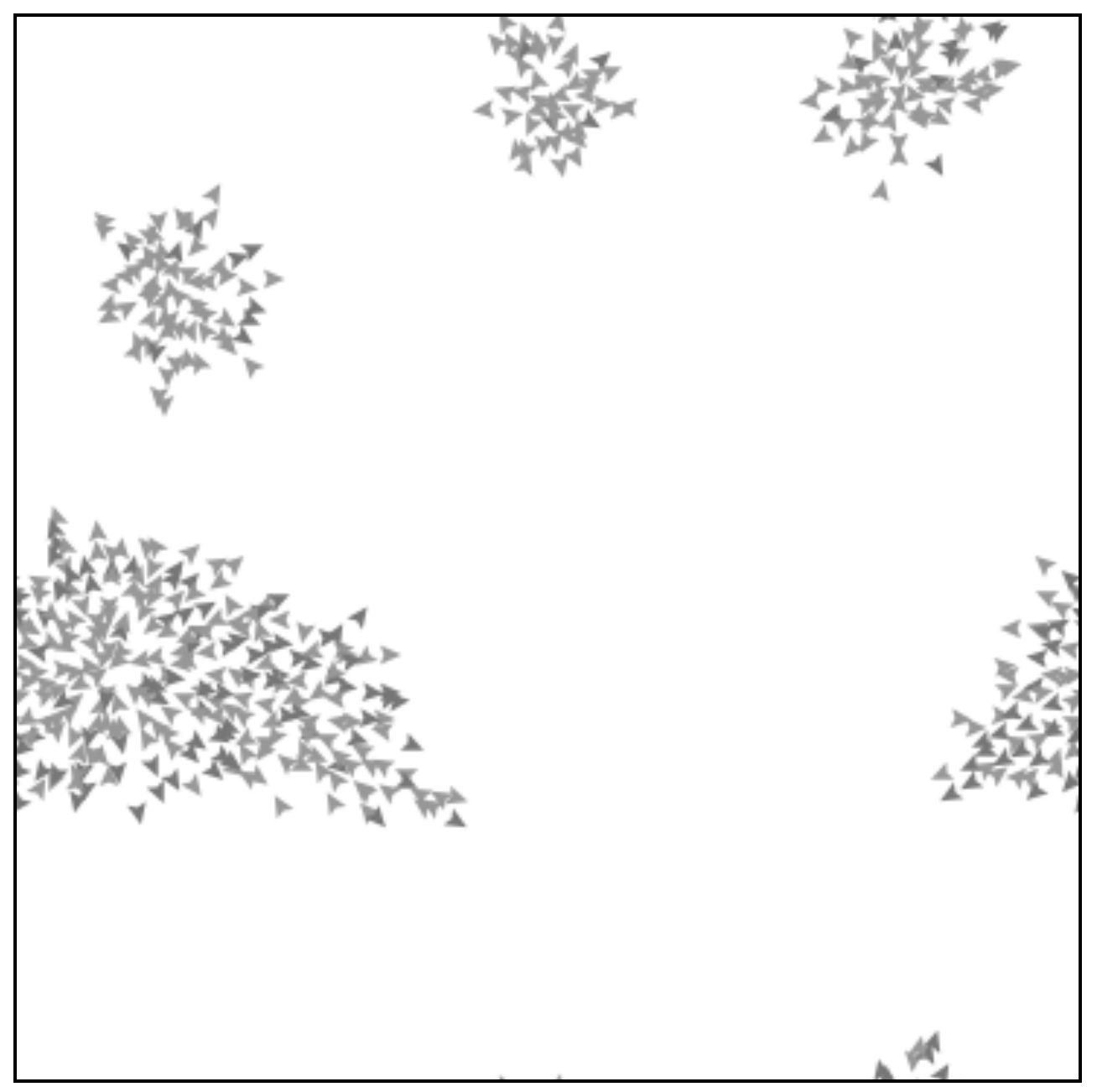

Figure 2: Snapshot of the simulation

Let us compare the behaviour of this model with the features of collectivities described in section 1 .

1. When we run the model, we see 'clumps', but drawing a boundary around clumps involves some arbitrary definition, perhaps in terms of local densities of agents.

2. While a definition of which agents are in and which out of a clump is possible (e.g. in terms of the distance to the nearest neighbour), again this seems arbitrary. If we treat the position of the agent as its location in 'knowledge space', then most agents within a 'clump' differ in their locations. 
3. Nevertheless, agents in the same clump are close together and so could be thought of as sharing some aspects of their knowledge.

4. The location of the clump, as measured for instance by its centroid, is constantly changing as some agents move more closely into the clump and others seek new less crowded locations.

5. Some agents consider themselves to be crowded and these behave differently from the other agents in the clump (by 'innovating' i.e. trying to find less crowded positions by moving through the knowledge space). These agents are located more centrally in the clumps and are influential in setting the direction of travel of the other agents.

\section{Conclusion}

The features of collectivities that we observe in society, summarised in section 1, thus emerge in the model as a result of the behaviour of the agents following the cycle of actions listed in section 4. While other micro-level actions could produce the same or similar macro-level patterns (Gilbert, 2002), it is useful to know that these do yield the macro-level behaviour that we observe. Specifically, we can conclude from the model that if:

1. agents change their ideas in knowledge-space in response to 'over-crowding' (Mulkay \& Turner, 1971);

2. some ideas and some agents are considered to be high status or important;

3. agents are motivated to copy and adopt those ideas or a variation on them;

then the phenomenon we have described as a 'collectivity' will emerge from the agents' behaviour.

There are many areas of social life where these micro-behaviours may be found and correspondingly many emergent collectivities. This paper has presented one generic model that can help to explain this wide range of social phenomena. The value of such generic models for so- 
ciology is twofold: they can account for the generation of particular phenomena, following Epstein's maxim that 'to explain macroscopic social patterns, we generate-or "grow"-them in agent models' (Epstein, 2005); and they can help to highlight commonalities and differences between phenomena which otherwise might be considered incomparable. For example, the present exercise raises the question, what are the significant similarities and differences between 'punks' and 'scientists', given that their social formations can be recreated using the same generic model? Devising, cataloguing and exploring the applicability of a wide range of generic models that are able to simulate many different social phenomena could be a productive research strategy to follow. Another potentially fruitful avenue for further research follows on from the observation ${ }^{1}$ that the model is a type of evolutionary model, in which there are processes of selection (in favour of those with high status), variation (through innovation) and adaptation (by movement through knowledge space). This opens up the possibility of studying the dynamics of collectivities by methods similar to those used to study natural selection, although one needs to respect the differences between biological evolution and social 'evolution' (for example, that there is no distinction between phenotype and genotype in the latter).

There are also many features of collectivities that the model has no ambitions to reproduce, but which might be considered in extensions or specialisations. For example, it says nothing about how collectivities are born, or how they die or fade away, or about the dynamics of these processes. It also leaves open questions about the form and dimensions of the knowledge space, and how these characteristics may vary between different collectivities. Details of these matters may not be appropriate for a generic model that tries to present an 'abstract social process' that recurs in many different forms in society, although they will be of great significance if one wants to study and understand a particular collectivity.

\footnotetext{
${ }^{1}$ By Petra Ahrweiler in response to the presentation of an earlier version of this paper at the ABModSim workshop
} 
Appendix: Pseudo-code of the model

Initialisation

Create agents and distribute them at random in knowledge space

\section{Execution}

Loop forever

Each agent:

Counts the number of other agents within its local-radius

Each agent:

Compares the number of other agents within its local-radius with the threshold

If the number is greater than the threshold

Then (the agent is crowded)

The agent locates that agent within visible-radius with the most agents surrounding it

The agent moves a distance proportional to speed towards this agent Else (the agent is lonely)

The agent locates the agent within visible-radius with the most agents surrounding it

The agent moves a distance proportional to speed away from this agent End loop 


\section{References}

Abbott, Andrew. (1995) Things of boundaries. Social Research 62(4) 857-82.

Ahrweiler, Petra, \& Gilbert, Nigel (Eds.). (1998). Computer Simulations in Science and Technology Studies. Berlin: Springer.

Ahrweiler, Petra, Pyka, Andreas, \& Gilbert, Nigel. (2004). Simulating Knowledge Dynamics in Innovation Networks (SKIN). In R. Leombruni \& M. Richiardi (Eds.), Industry and Labor Dynamics: The Agent-based Computational Economics Approach. Singapore: World Scientific Press.

Elias, Norbert (1939/1969) The Civilising Process. Oxford: Blackwell.

Epstein, Joshua M. (2005) Remarks on the Foundations of Agent-Based Generative Social Science. CSED Working Paper No. 41. The Brookings Institution, Washington, DC.

http://www.brookings.edu/es/dynamics/papers/CSED_wp41.pdf

Gilbert, Nigel. (1997). A simulation of the structure of academic science. Sociological Research Online, 2(2), < $\underline{\mathrm{http}}$ ://www.socresonline.org.uk/socresonline/2/2/3.html.

Gilbert, Nigel. (2002). Varieties of emergence. Paper presented at the Agent 2002 Conference: Social agents: ecology, exchange, and evolution, Chicago http://www.complexityscience.net/archive/00000001/.

Goolsby, Rebecca (2006) Combating terrorist networks: an evolutionary approach. Computational and Mathematical Organization Theory. 12: 7- 20.

Hodkinson, P. (2002), Goth Identity, Style and Subculture, Oxford, Berg

Mulkay, M.J., \& Turner, B.S. (1971). Over-production of Personnel and Innovation in three social settings. Sociology, 5(1), 47-61. 
O' Sullivan, David, \& Macgill, James. (2005). Modelling urban residential neighbourhood dynamics. Paper presented at the Modelling Urban Social Dynamics, University of Surrey, Guildford, UK.

Reynolds, C. W. (1987). Flocks, Herds, and Schools: A Distributed Behavioral Model. Computer Graphics, 21(4), 25-34.

Slanina, Franti`sek. (2000). Self organization in the Minority Game model. arXiv:condmat/0006098.

Thornton, Sarah (1995). Club cultures: music, media and subcultural capital. Cambridge: Polity.

Widdicombe S. and Wooffitt R.C. (1990) "Being" versus "doing" punk (etc): on achieving authenticity as a member. Journal of Language and Social Psychology, 9, 257-277.

Wittgenstein, Ludwig, \& Anscombe, G. E. M. (1953). Philosophical investigations. Oxford: Basil Blackwell.

\section{Footnote}

